\title{
Papel do cirurgião-dentista nas visitas domiciliares: atenção em saúde bucal
}

\author{
Role of surgeon-dentists in home visits: oral health care

\section{Papel del dentista en las visitas domiciliarias: atención en salud bucal}

Davi Oliveira Bizerril. Programa de Pós-Graduação em Odontologia da Universidade Federal do Ceará (UFC).

Fortaleza, CE, Brasil. davibizerril@gmail.com. (Autor correspondente)

Kátia de Góis Holanda Saldanha. Programa de Pós-Graduação em Odontologia da Universidade Federal do Ceará (UFC).

Fortaleza, CE, Brasil. katiasaldanha@ terra.com.br

Joacy Parente da Silva. Prefeitura Municipal de Fortaleza (PMF). Fortaleza, CE, Brasil. jpsilva@ netbandalarga.com.br

Janaína Rocha de Sousa Almeida. Programa de Pós-Graduação em Odontologia da Universidade Federal do Ceará (UFC).

Fortaleza, CE, Brasil. drajanainarocha@ hotmail.com

Maria Eneide Leitão Almeida. Programa de Pós-Graduação em Odontologia da Universidade Federal do Ceará (UFC).

Fortaleza, CE, Brasil. eneideufc@ hotmail.com

\section{Resumo}

Este artigo relata a experiência de cirurgiões-dentistas que realizam visitas domiciliares como uma forma de garantir o acesso à saúde bucal de uma parcela da população incapacitada de chegar à Unidade de Atenção Primária à Saúde. Tem como objetivo identificar ações e atividades desenvolvidas pelo cirurgião-dentista nas visitas domiciliares. A experiência ocorreu em domicílios de usuários da Unidade de Atenção Primária à Saúde José Valdevino de Carvalho no Município de Fortaleza, capital do Ceará, no período de janeiro a novembro de 2013. As ações e atividades identificadas foram categorizadas nos níveis básicos de atenção à saúde: promoção, prevenção, investigação de lesões de tecidos moles e limitações de danos. Os resultados mostraram que a frequência das atividades de promoção e prevenção foi alta. A frequência de ações para investigar lesões de tecidos moles e de limitações de danos de problemas bucais préexistentes foi baixa. Os dentistas realizaram 96 visitas domiciliares no propósito de oferecer uma cobertura total aos usuários incapacitados de locomoção. As ações e atividade de promoção em saúde basearam-se no estímulo à prática de hábitos saudáveis e orientações sobre tabagismo e alcoolismo. As ações de prevenção foram instrução de higiene oral, escovação supervisionada e aplicação tópica de flúor. As demais ações para limitar danos de problemas bucais já pré-existentes foram extrações de dentes com problemas periodontais. Desta maneira, o cirurgião-dentista nas visitas domiciliares assume um importante papel de facilitador para o bem-estar físico e social dos usuários impossibilitados de ir até uma Unidade de Atenção Primária à Saúde.

\section{Abstract}

This article reports the experience of dentists who performed home visits as a way to ensure access to the oral health among a portion of the population that was unable to reach primary health care units. This study aimed to identify the actions and activities developed by dentists for home visits. The experience occurred in the residences of users of the primary health care unit José Valdevino de Carvalho, in the city of Fortaleza, capital of Ceará, from January to November 2013. The actions and activities identified were categorized into the following basic levels of health care: promotion, prevention, investigation of soft tissue lesions and limitation of damages. The results showed that the frequency of activities for promotion and prevention was high. However, the frequency of actions for investigating soft tissue lesions and for limiting damages due to existing oral problems was low. The dentists performed 96 home visits in order to offer total coverage to disabled users of locomotion. The actions and health promotion activities were based on encouraging healthy habits and practice guidelines on smoking and alcoholism. Preventive actions were oral hygiene instruction, supervised brushing and topical application of fluoride. The other actions for limiting damage due to pre-existing oral problems were teeth extractions in individuals with periodontal problems. Thus, dentists who perform home visits assume an important role that facilitates the physical and social well-being of individuals who are unable to go to a primary health care unit.

Como citar: Bizerril DO, Saldanha KGH, Silva JP, Almeida JRS, Almeida MEL. Papel do cirurgião-dentista nas visitas domiciliares: atenção em saúde bucal. Rev Bras Med Fam Comunidade. 2015;10(37)1-8. http://dx.doi.org/10.5712/rbmfc10(37)1020
Palavras-chave:

Visita Domiciliar

Saúde Bucal

Humanização da Assistência Dentistas

\section{Keywords:}

Home Visit

Oral Health

Humanization of Assistance Dentists

Fonte de financiamento:

declaram não haver.

Parecer CEP:

não se aplica.

Conflito de interesses: declaram não haver.

Procedência e revisão por pares: revisado por pares.

Recebido em: 06/10/2014. Aprovado em: 03/08/2015. 


\section{Resumen}

En este artículo se describe la experiencia de los dentistas que hacen visitas a domicilio como una forma de garantizar el acceso a la salud oral de una parte de la población incapaz de llegar a la Unidad de Atención Primaria a la Salud. Tiene como objetivo identificar las acciones y actividades de los dentistas en las visitas domiciliarias. El experimento se llevó a cabo en los hogares de los miembros de la Unidad de Atención Primaria de Salud José Valdevino de Carvalho, en Fortaleza, capital de Ceará, en el período entre enero y noviembre del 2013. Las acciones y actividades identificadas se clasifican en los niveles básicos de atención a la salud: promoción, prevención, investigación de lesiones de tejidos blandos y limitaciones de daños. Los resultados mostraron que la frecuencia de las actividades de promoción y prevención ha sido alta. La frecuencia de las acciones para investigar las lesiones de tejidos blandos y limitaciones de daños de los problemas dentales pre-existentes fue baja. Los dentistas realizaron 96 visitas a los hogares con el fin de proporcionar una cobertura completa para los usuarios con discapacidad de locomoción. Las acciones y actividades de promoción a la salud se basan en estímulos para la práctica de hábitos saludables y pautas sobre el tabaquismo y el alcoholismo. Las acciones de prevención eran instrucciones de higiene oral, bajo la supervisión de cepillado y aplicación de flúor tópico. Otras acciones para limitar los daños pre-existentes eran extracciones de dientes con problemas periodontales. Por lo tanto, las visitas a domicilio dentista asume un papel de facilitador para el bienestar físico y social de los usuarios que no pueden ir a una Unidad de Atención Primaria de Salud.
Palabras clave:

Visita Domiciliaria

Salud Bucal

Humanización de la Atención Odontólogos

\section{Introdução}

A atenção domiciliar (AD) é caracterizada por um conjunto de ações e serviços de promoção à saúde, prevenção e tratamento de doenças e reabilitação prestadas em domicílio, com garantia de continuidade de cuidados, além de ser integrada às redes de atenção à saúde. ${ }^{1}$

Este tipo de atenção vem designar a "desospitalização" do cuidado, aproximando o usuário do profissional. É considerada um componente do continuum dos cuidados à saúde, pois os serviços de saúde são oferecidos ao indivíduo e sua família em seus domicílios com o propósito de: promover, manter ou restaurar a saúde; maximizar o nível de independência; minimizando os efeitos das incapacidades ou doenças, incluindo aquelas sem perspectiva de cura. ${ }^{2,3}$

A AD é um processo integrado pelo atendimento, a visita e a internação em domicílio. Então, uma das partes que compõem esta atenção é a visita domiciliar realizada por profissionais a fim de prestar uma atenção em saúde permeada de vínculo e humanização.

A visita domiciliar apresenta-se como uma forma de acesso ao usuário às ações e serviços de saúde da Estratégia Saúde da Família (ESF). Esta atividade se caracteriza pela visita das equipes de saúde da família e de saúde bucal ao domicílio dos usuários assistidos, com o objetivo de reconhecer o ambiente familiar diagnosticando os nós críticos pertinentes à realidade das famílias, para que, posteriormente, possa servir de subsídios para um adequado planejamento de ações em saúde, além de recuperar os indivíduos necessitados. . $^{4,5}$

Os princípios básicos, éticos e doutrinários do Sistema Único de Saúde (SUS) são obedecidos na visita domiciliar como: a integralidade, oferecendo atendimento ao usuário dentro dos três níveis de atenção; a universalidade, inserindo o usuário incapacitado de se locomover à unidade de saúde para atendimento; e a equidade, atendendo às necessidades dos que mais precisam de cuidados em saúde. Ressalta-se, ainda, o acolhimento, que é a humanização no atendimento, dando direito ao usuário em ser escutado, de forma qualificada e singular. Todos estes princípios fortalecem o vínculo entre a população usuária e o serviço de saúde. ${ }^{4,6-10}$

Assim como o médico e o enfermeiro, o cirurgião-dentista (CD) da ESF tem, também, como competência realizar visitas domiciliares no propósito de oferecer atenção em saúde bucal individual e coletiva às famílias, ${ }^{11}$ além de contribuir para a promoção e prevenção em saúde. 
Diversas ações são realizadas no domicílio como, por exemplo, a busca ativa de lesões bucais, ações de vigilância em saúde bucal e de educação em saúde bucal, as quais o cirurgião deverá participar como componente ativo da equipe Saúde da Família. ${ }^{4}$

Este artigo tem como objetivo geral identificar ações e atividades desenvolvidas pelo cirurgião-dentista nas visitas domiciliares.

\section{Descrição da experiência}

O referido trabalho se desenvolveu em domicílios assistidos pela unidade de atenção primária à saúde (UAPS) José Valdevino de Carvalho, da Secretaria Regional IV do município de Fortaleza, no período de julho a novembro de 2013.

A unidade situa-se no bairro Itaóca cobrindo uma área de 15.248 habitantes, aproximadamente 4.455 famílias $^{12}$. Da população total assistida, $85 \%$ são provenientes do próprio bairro e $15 \%$ é constituída pela população do bairro Parangaba.

A UAPS José Valdevino de Carvalho tem quatro equipes de Saúde da Família (EqSF) contendo quatro médicos generalistas, quatro cirurgiões-dentistas e quatro enfermeiros. O recurso humano auxiliar conta com 1 auxiliar em saúde bucal (ASB), 2 técnicos em saúde bucal (TSB), 4 técnicas de enfermagem e 18 agentes comunitários de saúde (ACS). Além das equipes de saúde da família e de saúde bucal, a UAPS possui um médico pediatra e um ginecologista.

A área coberta é considerada de risco psicossocial com pontos de drogas e áreas de conflitos (violência entre gangues). Não está dentro dos critérios de risco ambiental, embora em alguns espaços ainda existam esgoto a céu aberto, áreas de invasão, dentre outros fatores agravantes.

No processo de reterritorialização, no início de 2013, foi constatada a existência de áreas descobertas, ou seja, áreas sem cadastros e não assistidas pelos agentes comunitários de saúde (ACS). A população destas áreas é atendida por meio de demanda livre, apesar do atendimento da UAPS ser caracterizado por demanda programada pelos ACS.

$O$ atendimento da UAPS é caracterizado por atendimento de demanda programada das áreas cobertas pelas EqSF e atendimento de demanda espontânea das áreas descobertas.

Este estudo caracteriza-se por ser um relato de caso, cujo objetivo geral é apresentar as ações e atividades desenvolvidas pelos cirurgiões-dentistas nas visitas domiciliares. Os objetivos específicos foram: conhecer as condições de vida e hábitos do usuário; identificar obstáculos no autocuidado em saúde bucal; oferecer atenção extraclínica ao usuário com dificuldade de locomoção; e detectar fatores de risco à saúde bucal.

O trabalho buscou de forma clara e objetiva, oferecer uma contribuição ao incremento da promoção e prevenção de saúde bucal pela motivação, educação e diagnóstico de doenças bucais em indivíduos incapacitados de comparecimento à UAPS por várias razões como envelhecimento, sequelas de enfermidades, portadores de limitações físicas, psíquicas e motoras.

Os dados da pesquisa foram organizados e analisados no programa de estatística SPSS 22.0 para Windows (SPSS Inc, Chicago, IL, USA).

Todos os aspectos éticos em pesquisa foram obedecidos como dignidade dos usuários, sigilo de identidade dos domiciliados e a não identificação de dados específicos de participantes. Além disso, os 
quatro princípios básicos da bioética foram seguidos: autonomia, justiça, beneficência e não maleficência. A pesquisa não ofereceu nenhum risco aos participantes.

\section{Discussão}

As visitas domiciliares foram realizadas por duas equipes de saúde bucal da UAPS, formada por dois CD e dois técnicos em saúde bucal (TSB), além do respectivo ACS da microárea.

Com as visitas, foi possível oferecer as equipes de saúde bucal o conhecimento da realidade de vida da população, favorecendo o estabelecimento de vínculos, e a compreensão dos hábitos e relações familiares daquela comunidade. As visitas visaram também à redução de custos por meio da substituição ou abreviação da internação hospitalar. ${ }^{3}$

As visitas contemplaram todas as microáreas cobertas por ACS, como mostra a Tabela 1. Foram priorizados os domicílios que tinham pacientes incapacitados de se locomover até a UAPS, como os acamados, idosos com dificuldades motoras, gestantes no final da gestação, dentre outras situações.

Foram realizadas 96 visitas domiciliares no propósito de oferecer uma cobertura total aos usuários incapacitados de locomoção.

No decorrer das visitas domiciliares foram realizados procedimentos em todos os níveis de atenção em saúde, de acordo com a necessidade dos usuários (Tabela 2).

Os profissionais cirurgiões-dentistas realizaram educação em saúde dentro do contexto do autocuidado em saúde da gestante, do recém-nascido (RN) e da puérpera, do idoso portador de deficiência motora e visual e de adultos acamados. ${ }^{13-15}$

\section{Saúde bucal em gestantes}

Em visitas domiciliares realizadas às gestantes, foi realizada a orientação sobre os cuidados de higiene oral das mães e dos bebês, já que o estado de saúde bucal da gestante pode influenciar na saúde geral e bucal do bebê. A cárie dentária e, principalmente, as doenças periodontais foram assuntos abordados pelos cirurgiões-dentistas pelo fato de influenciarem na antecipação do trabalho de parto e nascimento de bebês prematuros. ${ }^{16}$ Visto a necessidade de atendimento odontológico, as gestantes foram encaminhadas à UAPS para o devido tratamento. $O$ trabalho interdisciplinar desenvolvido pela equipe de saúde da família deve ser priorizado para levar uma atenção integral à saúde da gestante e do bebê. Então, o trabalho do cirurgião-dentista perpassa todo o pré-natal da gestante, na UAPS e no domicílio, mostrando-se complementar ao processo de trabalho do enfermeiro e médico.

Tabela 1. Distribuição da frequência absoluta de famílias assistidas e usuários limitados à locomoção segundo as EqSF da UPAS José Valdevino de Carvalho. Fortaleza. Ceará, 2013.

\begin{tabular}{ccccc} 
EqSF & \multicolumn{2}{c}{ Famílias assistidas } & \multicolumn{2}{c}{ Usuários limitados à locomoção } \\
& $\mathbf{n}$ & $\%$ & $\mathbf{n}$ & 20 \\
I & 260 & 11,11 & 23 & 26,08 \\
II & 1011 & 43,20 & 30 & 36,52 \\
III & 564 & 24,10 & 42 & 17,39 \\
IV & 505 & 21,58 & 20 & 100 \\
TOTAL & 2340 & 100 & 115 & 100 \\
\hline
\end{tabular}

Fonte: própria pesquisa, 2014. 
Tabela 2. Distribuição da frequência absoluta e percentual das atividades desenvolvidas em visitas domiciliares, de acordo com o nível de atenção à saúde/saúde bucal. Fortaleza. Ceará, 2013.

\begin{tabular}{|c|c|c|c|}
\hline $\begin{array}{l}\text { Nível de atenção à } \\
\text { saúde/saúde bucal }\end{array}$ & Atividades desenvolvidas em visita domiciliar & $\begin{array}{l}\text { Frequência Absoluta de } \\
\text { atividades realizadas (n) }\end{array}$ & $\begin{array}{l}\text { Percentual de atividades } \\
\text { realizadas }(\%)\end{array}$ \\
\hline \multirow{2}{*}{ Promoção } & Busca de hábitos saudáveis & 92 & 18,66 \\
\hline & Desestímulo ao alcoolismo e tabagismo & 13 & 2,63 \\
\hline \multirow{4}{*}{ Prevenção } & Orientação e instrução de higiene oral & 96 & 19,47 \\
\hline & $\begin{array}{l}\text { Distribuição de kits odontológicos (escova dental, } \\
\text { creme dental e fio dental) }\end{array}$ & 96 & 19,47 \\
\hline & Aplicações tópicas de flúor & 56 & 11,35 \\
\hline & Escovação supervisionada & 96 & 19,47 \\
\hline \multirow{5}{*}{ Recuperação } & Anamnese & 11 & 2,23 \\
\hline & Exames clínicos & 6 & 1,22 \\
\hline & Prescrições terapêuticas & 7 & 1,42 \\
\hline & $\begin{array}{l}\text { Exodontias de restos radiculares e de dentes } \\
\text { comprometidos gravemente por doença periodontal }\end{array}$ & 5 & 1,02 \\
\hline & Remoção de suturas & 3 & 0,60 \\
\hline $\begin{array}{l}\text { Matriciamento de } \\
\text { diagnóstico bucal }\end{array}$ & Busca e diagnóstico de lesões bucais & 12 & 2,43 \\
\hline TOTAL & & 493 & 100 \\
\hline
\end{tabular}

Fonte: própria pesquisa, 2014.

O cirurgião-dentista, também, realiza a escuta qualificada, incluindo o acolhimento dos anseios, crenças e tabus das gestantes. Orienta, com respeito, possíveis mudanças na cavidade bucal durante a gravidez, ressaltando as maneiras adequadas de ter um autocuidado corporal, hábitos de vida saudável e uma boa saúde bucal.

\section{Saúde bucal na puericultura}

A puericultura é muito mais além de um conjunto de técnicas de higiene, nutrição e cuidado com a criança. O acolhimento deve estar dentro deste processo como forma de reforçar o vínculo e a humanização, principalmente, entre o cirurgião-dentista e o usuário.

Nos domicílios das puérperas foram realizadas orientações e instruções de higiene oral materna e, principalmente, infantil. Os cirurgiões-dentistas demonstraram para as puérperas a forma adequada de higienização oral dos bebês com a utilização de fraldas umedecidas com água filtrada para a limpeza pós-mama dos roletes gengivais. ${ }^{17,18}$ Além disso, alguns bebês foram submetidos a exame clínico para a verificação da presença de dentes neonatais, assim como anormalidades como a presença de fendas palatais, anquiloglossia parcial/total ou cistos orais palpáveis.

Informações foram fornecidas às mães sobre a importância da amamentação no desenvolvimento do sistema estomatognático, evitando um futuro respirador bucal e ortodôntico. ${ }^{18}$ Também foram ressaltados os valores de benefícios imunológicos, psicossociais e alimentares no processo de amamentação. Desta maneira, a amamentação deve ser mencionada com um dos principais fatores que favorecem ao fortalecimento do vínculo entre mãe e filho.

\section{Saúde bucal em pacientes acamados}

Nas residências de usuários acamados, por diversos motivos como idosos com idade avançada e jovens adultos vítimas de violência urbana e acidentes de transporte terrestre, foram realizadas orientações 
sobre qualidade de vida como a criação de artefatos para evitar escoriações nas costas pelo fato do grande tempo em decúbito dorsal e a realização de arteterapias como desenhos e pinturas. Foram realizadas ações de escovação dentária e o uso de fio dental acompanhadas de orientações sobre higienização bucal. ${ }^{19}$

Nos pacientes com diagnóstico de câncer bucal, realizado por meio do exame clínico, a visita domiciliar fornecia o apoio psicológico-afetivo do paciente por parte da equipe de saúde bucal, o que ajudava o paciente no enfrentamento dos processos de terapia, tais como, quimioterapia e, principalmente, de radioterapia por ser extremamente dolorosa e que incapacitava a alimentação do paciente. Além disso, a visita favorecia o acompanhamento clínico das lesões por parte do cirurgião-dentista.

Nos domicílios de idosos dependentes e semidependentes foram desenvolvidas ações de promoção e prevenção em saúde como orientações sobre a saúde sistêmica incluindo doença como hipertensão arterial, diabetes mellitus e Alzheimer, além de estímulos a mudanças de hábitos sedentários para rotinas de vida saudável como a realização de caminhadas e visitas frequentes ao médico e cirurgião-dentista. Quanto à saúde bucal, foram dadas orientações sobre medidas de higienização bucal incluindo métodos de escovação dentária e protética, alterações de tecidos bucais e possíveis lesões orais. ${ }^{20-23}$

Quanto aos pacientes com necessidades especiais, por diversos motivos mentais e patológicos como os sequelados de acidente vascular cerebral (AVC), foram realizadas instruções de cuidado na

saúde bucal levando em consideração os conhecimentos da família e dos cuidados quanto à doença. ${ }^{24,25}$ Os familiares e cuidadores devem receber uma atenção especial - educação em saúde e estímulo de força - na colaboração no cuidado a estes usuários acamados.

\section{Visitas domiciliares}

Pode-se apontar, assim como Barros et al., ${ }^{26}$ que as visitas domiciliares são benéficas, trazendo resultados positivos, pois é uma estratégia que viabiliza atender àqueles que possuem necessidades especiais, como idosos com idade bastante avançada, acamados como vítimas de acidentes e violência urbana, usuários com necessidades especiais como deficiência mental e motora. Para estes pacientes, há vários obstáculos devido à própria condição do paciente no acesso aos serviços de saúde odontológicos ofertados dificultando sua assistência em saúde bucal.

Existem vários nós críticos no processo de realização de visitas domiciliares como a dificuldade de acesso aos domicílios por inúmeros motivos como malha viária urbana precária, violência urbana, tráfico de drogas e falta de transporte. Há, ainda, outros fatores como a recusa de profissionais de saúde e agentes comunitários de saúde a realizar tal atividade, priorização da assistência à saúde na UAPS pela gestão municipal, entre outros.

Corroborando com o Ministério da Saúde, o cirurgião-dentista apresenta papel fundamental na visita domiciliar desenvolvendo atividades de: promoção em saúde por meio da motivação e educação em saúde para o paciente e sua família; proteção e prevenção em saúde bucal como orientar higiene bucal e cuidados com prótese ao paciente, aplicação tópica de flúor e escovação supervisionada; tratamento clínico por meio de identificação de lesões orais. Além disso, o profissional estabelece uma rede de comunicação participativa com a família, coordenando o cuidado do paciente acamado com a saúde familiar. ${ }^{4}$

Como metas alcançadas, podem-se citar: o acesso aos usuários incapacitados de se dirigirem à UAPS (por vários motivos como barreiras físicas, socioeconômicas e de operacionalização dos serviços 
de saúde); a rapidez no processo de cura com cuidado continuado no domicílio; a minimização de intercorrências clínicas; a diminuição de riscos de infecções hospitalares por oferecer atendimento odontológico no domicílio; a minimização dos efeitos das incapacidades ou doenças; o incentivo à aproximação profissional-paciente favorecendo o vínculo, desmistificando a figura do profissional da saúde como um "deus".

Há, ainda, como resultados esperados, o suporte emocional para o paciente e familiares; a instituição do papel do cuidado perante a situação do usuário; o oferecimento da autonomia para o paciente, família e cuidador no cuidado em saúde; a orientação e instrução de pacientes e de seus cuidadores; o oferecimento de uma melhor qualidade, no seu contexto de doenças incuráveis, ao paciente terminal; a realização de procedimentos curativistas devolvendo a saúde bucal para estes pacientes.

\section{Conclusão}

É notório que a visita domiciliar ainda não está incorporada às atividades da equipe de saúde bucal da estratégia de saúde da família, porém ressalta-se que o cirurgião-dentista e toda a equipe de saúde bucal devem inseri-la no seu cotidiano para o estabelecimento de um vínculo mais efetivo entre a equipe de saúde bucal e a comunidade assistida, tendo como consequência uma maior humanização da atenção e do cuidado em saúde bucal.

A visita domiciliar proporciona a equidade e um atendimento humanizado a uma parcela da população distanciada da odontologia tradicional reconhecendo suas diferenças.

Este estudo ressalta a importância do cirurgião-dentista da ESF nas visitas domiciliares, contribuindo para o bem-estar físico e social dos pacientes. As principais atividades desenvolvidas nas visitas domiciliares realizadas por cirurgiões-dentistas são aquelas voltadas para promoção e prevenção em saúde bucal.

\section{Referências}

1. Brasil. Ministério da Saúde. Portaria no 2.527 de 27 de outubro de 2011. Redefine a Atenção Domiciliar no âmbito do Sistema Único de Saúde (SUS). Brasília: Diário Oficial da União; 2011.

2. Marrelli TM. Welcome to home care: the health setting of the future. In: Marrelli TM, editor. Handbook of Home Health Orientation. Saint Louis: Mosby; 1997.

3. Giacomozzi CM, Lacerda MR. A Prática da Assistência Domiciliar dos Profissionais da Estratégia de Saúde da Família. Texto Contexto Enferm. 2006;15(4):645-53. DOI: http://dx.doi.org/10.1590/S0104-07072006000400013

4. Brasil. Ministério da Saúde. Secretaria de Atenção à Saúde. Departamento de Atenção Básica. Caderno de Atenção Domiciliar - Volume 1. Capítulo 1 - Atenção Domiciliar e o SUS. Brasília: Ministério da Saúde; 2012.

5. Mendes AO, Oliveira FA. Visitas domiciliares pela equipe de Saúde da Família: reflexões para um olhar ampliado do profissional. Rev Bras Med Fam Comunidade. 2007;2(8):253-60.

6. Campos CR. A produção da cidadania - construindo o SUS em Belo Horizonte. In: Campos CR, Malta DC, Reis AT, Santos AF, Merhy EE, orgs. Sistema Único de Saúde em Belo Horizonte: reescrevendo o público. São Paulo: Xamã; 1998. p.11-30.

7. Merhy EE. Em busca da qualidade dos serviços de saúde: os serviços de porta aberta para a saúde e o modelo tecnoassistencial em defesa da vida. In: Cecílio L, org. Inventando a mudança na saúde. São Paulo: Hucitec; 1994. p.117-60.

8. Merhy EE. Em busca do tempo perdido: a micropolítica do trabalho vivo em saúde. In: Merhy EE, Onocko R, orgs. Agir em saúde: um desafio para o público. São Paulo: Hucitec; 1997. p.71-112.

9. Malta DC, Merhy EE. Buscando novas modelagens em saúde: as contribuições do Projeto Vida e do acolhimento para a mudança do processo de trabalho na rede pública de Belo Horizonte, 1993 - 1996. In: Brasil. Ministério da Saúde. Experiências inovadoras no SUS: produção científica doutorado e mestrado. Brasília: Ministério da Saúde;2002. p. 69-101. 
10. Solla JJSP. Acolhimento no sistema municipal de saúde. Rev Bras Saúde Mater Infant. 2005;5(4):493-503. DOI: http:// dx.doi.org/10.1590/S1519-38292005000400013

11. Brasil. Ministério da Saúde, Secretaria de Atenção à Saúde, Departamento de Atenção Básica. Política Nacional de Atenção Básica. Brasília: Ministério da Saúde; 2012.

12. Instituto de Pesquisa e Estratégia Econômica do Ceará (IPECE). Ceará em mapas. Caracterização territorial: limites e regionalizações. 2007 [Acesso 16 Dez 2013]. Disponível em: http://www2.ipece.ce.gov.br/atlas/capitulo1/11/140x.htm.

13. Martins JJ, Albuquerque GL, Nascimento ERP, Barra DCC, Souza WGA, Pacheco, WNS. Necessidades de educação em saúde dos cuidadores de pessoas idosas no domicílio. Texto Contexto Enferm. 2007;16(2):254-62. DOI: http://dx.doi. org/10.1590/S0104-07072007000200007

14. Souza LM, Wegner W, Gorini, MIPC. Educação em Saúde: uma estratégia de cuidado ao cuidador leigo. Rev Latino-am Enfermagem. 2007;15(2):337-43. DOI: http://dx. doi.org/10.1590/S0104-11692007000200022

15. Chiesa AM, Veríssimo MDLOR. A Educação em Saúde na Prática do PSF. In: Instituto para o Desenvolvimento da Saúde (IDS); Universidade de São Paulo (USP); Ministério da Saúde, eds. Manual de Enfermagem. Brasília: Ministério da Saúde; 2001 [acesso 01 Maio 2014]. p.34-42. Disponível em: http://intranet.ftc.br/upload/141196/195508_A_Educa_o_em_Sa_ de_na_Pr_tica_do_PSF.pdf.

16. Cruz SS, Costa MCN, Gomes Filho, IS, Vianna MIP, Santos CT. Doença periodontal materna como fator associado ao baixo peso ao nascer. Rev Saúde Pública. 2005;39(5):782-7. DOI: http://dx.doi.org/10.1590/S0034-89102005000500013

17. Cruz AAG, Gadelha CGF, Cavalcanti AL, Medeiros PFV. Percepção materna sobre a higiene bucal de bebês: um estudo no Hospital Alcides Carneiro, Campina Grande-PB. Pesq Bras Odontoped Clin Integr. 2004;4(3):185-9.

18. Simioni LRG, Comiotto MS, Rêgo DM. Percepções maternas sobre a saúde bucal de bebês: da informação à ação. RPG Rev Pos-Grad. 2005;12(2):167-73.

19. Coelho FP. Visita domiciliar e o cuidado da saúde bucal dos pacientes acamados. Anais do 12 o Congresso Brasileiro de Medicina da Família e Comunidade. 2013, 30 de Maio a 2 de Junho; Belém, PA.

20. Hiramatsu DA, Tomita NE, Franco LJ. Perda dentária e a imagem do cirurgião-dentista entre um grupo de idosos. Ciênc Saúde Coletiva. 2007;12(4):1051-6. DOI: http://dx.doi.org/10.1590/S1413-81232007000400026

21. Miranda AF, Montenegro FLB. O cirurgião-dentista como parte integrante da equipe multidisciplinar direcionada à população idosa dependente no ambiente domiciliar. Rev Paul Odontol. 2009;31(3):15-9.

22. Braga EC, Sinatra LS, Carvalho DR, Cruvinel VR, Miranda AF, Montenegro FLB. Intervenção odontológica domiciliar em paciente idoso cego institucionalizado: relato de caso. Rev Paul Odontol. 2011;33(2):17-22.

23. Rocha DA, Franco MA. Atendimento odontológico domiciliar aos idosos: uma necessidade na prática multidisciplinar em saúde: revisão de literatura. Rev Bras Geriatr Gerontol. 2013;16(1):181-9. DOI: http://dx.doi.org/10.1590/S180998232013000100018

24. Tomita NE, Fagote BF. Programa Educativo em Saúde Bucal para Pacientes Especiais. Odontol Soc. 1999;1(1/2):45-50.

25. Chagas NR, Monteiro, ARM. Educação em saúde e família: o cuidado ao paciente, vítima de acidente vascular cerebral. Acta Scient Health Sci. 2004;26(1):193-204.

26. Barros GB, Cruz JPP, Santos AM, Rodrigues AAAO, Bastos KF. Saúde bucal a usuários com necessidades especiais: visita domiciliar como estratégia no cuidado à saúde. Rev Saúde Com. 2006;2(1):127-34. 